\title{
Autonomous Behavior Intelligence Control of Self-Evolution Mobile Robot for High-Voltage Transmission Line in Complex Smart Grid
}

\author{
Wei Jiang $\mathbb{D}^{1,2}$ Gan Zuo, ${ }^{1}$ De Hua Zou, ${ }^{3}$ Hongjun Li $\mathbb{D},{ }^{1,4}$ Jiu Jiang Yan, ${ }^{1}$ and Gao Cheng Ye \\ ${ }^{1}$ School of Mechanical Engineering and Automation, Wuhan Textile University, Wuhan 430073, China \\ ${ }^{2}$ Wuhan Textile University, Hubei Key Laboratory of Digital Textile Equipment, Wuhan 430200, China \\ ${ }^{3}$ State Grid Hunan Transmission Maintenance Company, Changsha 410100, China \\ ${ }^{4}$ College of Engineering, Huazhong Agricultural University, Wuhan 430070, China
}

Correspondence should be addressed to Wei Jiang; jiangwei2013@whu.edu.cn and Hongjun Li; lihongjun@wtu.edu.cn

Received 6 August 2020; Revised 11 September 2020; Accepted 9 October 2020; Published 5 November 2020

Academic Editor: Ruoli Tang

Copyright (c) 2020 Wei Jiang et al. This is an open access article distributed under the Creative Commons Attribution License, which permits unrestricted use, distribution, and reproduction in any medium, provided the original work is properly cited.

In complex smart grid, the power maintenance robot is important equipment to ensure the reliable operation of high-voltage lines and it is a useful exploration to realize high-quality power transmission. In view of the increasingly prominent contradiction between the robot single operation function and the diversification of power grid maintenance operations, additional with the robot weak autonomous operation and intelligent behavior ability, this paper proposes a new configuration of a reconfigurable power robot with terminal functions and its autonomous operation behavior control method for the three typical tasks which are the high-voltage transmission line insulators, drainage plates, and dampers maintenance. Through the analysis and planning of the robot operation behavior, the robot finite state machine (FSM) model in the three operation states has been established. Through the introduction of the state transfer function in the FSM, the automatic switching control between the robot key operation states can be realized, and the robot motion planning can be optimized. The movement and working flow of the robot improve the robot operation intelligence and operation efficiency. Based on this, the robot autonomous operation control system has been designed and the robot physical prototype has been developed for three maintenance tasks of insulators, drainage plates, and dampers. Finally, simulation experiments and field operation tests verify the effectiveness and engineering practicability of the proposed method. Compared with traditional manual control, the autonomous behavior control method can significantly improve the robot operational efficiency and operational intelligence. At the same time, the robot multitask function and autonomous behavior control under different tasks can be realized and the method has strong versatility for different task objects and different line environments. The research and its promotion have important theoretical significance and practical application value for the power system operation and maintenance integration management.

\section{Introduction}

Electricity is the lifeblood of the national economy and highvoltage cables are an important channel for power transmission. Their special geographical environment and harsh natural environment will cause various faults on line, in order to ensure the safe, normal, and stable operation of highvoltage transmission lines, thereby, effectively reducing economic losses. It is necessary to regularly and irregularly perform maintenance and construction operations on line fittings and their operation environment [1-4]. At present, such special operations in dangerous and harsh environments are all performed manually, which not only is labor-intensive and low-efficient but also poses great personal safety risks. Regarding the maintenance operations far away from the poles and towers, the live working can be performed only in the condition of power outages. As the assessment indicators of power transmission quality and operation safety are getting higher and higher, as well as the urgent needs of modern power system operation and management automation, the 
contradiction between this manual operation method and modern high-quality power transmission has become more and more prominent. Therefore, it is an effective measure to replace the live line operation performed by the live line maintenance robot [5-8], which has important practical application value for improving operation efficiency, operation reliability, and worker safety. The robots currently studied are mostly only oriented to double operations $[9,10]$, and most of them are only oriented to a single operation. Due to the wide variety and dispersion of line maintenance operations, power grid companies will inevitably need to configure robots of different types of operations, which result in high purchase and maintenance costs. Namely, the contradiction between the robot single operation function and the diversification of the power grid maintenance operation objects in the existing research has become more and more prominent $[11,12]$. Therefore, the study of multitask-oriented power maintenance robots and their autonomous operation behavior control so as to realize the multipurpose function of the robot mobile platform has important theoretical significance and practical value for building a resource-saving and environment-friendly integrated operation of the power transmission grid. In order to improve the robot autonomous behavior ability and operation efficiency, the robot is required to have a strong autonomous behavior control ability. In terms of autonomous behavioral control, there are mainly autonomous behavior control methods based on expert systems $[13,14]$, fuzzy logic $[15,16]$, neural network $[17,18]$, swarm intelligence algorithm $[19,20]$ and, additionally, the hierarchical planning method $[21,22]$ which have already been adopted to the power line inspection robot through using generative reasoner to create behavior sequences online at the behavior planning layer, it combined with the behavior interpretation knowledge base generated offline at the action layer, and to achieve automatic robot obstacle crossing on power line. A finite state machine model [23, 24] has been proposed and used in inspection robot obstacle crossing on HVTL; however, this method can only realize the robot's local obstacle crossing and semiautomatic obstacle crossing. In [25-28], a multisensor-based transmission line identification and robot spatial posture positioning method has been proposed; however, it did not perform multisensor information fusion processing, thereby affecting the improvement of the overall robot operation efficiency. Through the above analysis, we can know, there is currently no universal robot autonomous behavior control method since the complicated robot operation can be abstracted into a series of state combinations from the operation beginning to the task completion; in this way, the complex operation process of the robot has been simplified and the key to the improvement of the robot autonomous behavior ability is the free switching between different joint states. Therefore, the goal of robot behavior planning for multitask tasks in the ultrahigh-voltage multisplit environment is to formalize model descriptions of different types of tasks and robot motion control behaviors in all layers. This facilitates the completion of automatic reason and decision control and achieves the purpose of the electric power line robot autonomous operation.
Based on the above analysis, this article is aimed at the multisplit multitask power transmission maintenance robot. Based on the analysis of the robot completion insulator replacement, drainage plate tightening, and damper replacement of three typical operations' motion planation, the design method of hierarchical control has been adopted and the robot motion behavior during the operation can be divided into a combination of multiple basic behaviors by defining the basic behavior of the robot arm joint motion. The finite state machine (FSM) model is used to realize the management and control of the combined behavior and the robot operation motion behavior has been decomposed. By selecting and setting a reasonable operation state transfer function, the robot three different operations have been planned and optimized for motion control, compared with the traditional manual control, and the operation status reduces the operation intensity of the operator and improves the operation efficiency. At the same time, for some special states that the robot may appear in the operation process, a mechanism for handling robot abnormal behavior of the robot is designed. Finally, the experimental verification of the feasibility and engineering practicability of the robot behavior control algorithm designed in this paper through simulation and field operation experiments are of great significance for accelerating the robot practical process.

\section{The Function Realization of Multitask Reconfigurable Robot System}

2.1. Mechanical Configuration Analysis and Synthesis of Multitask Robots. Through the analysis of the three operation tasks and operation principles of insulator (auxiliary) replacement [29], drainage plate bolt tightening [30], and damper replacement [31], the three operation tasks can be completed, respectively. The degrees of freedom required by the robot manipulator and their corresponding functions are shown in Tables $1-3$.

It can be concluded from Tables 1-3 that in order to complete the three tasks, the number of DOF and the functions required by the robot dual operation arms are completely the same. The only difference is the DOF and functions of the manipulator. Therefore, the live maintenance robot can be designed using the configuration mode of the robot mobile platform and the manipulator reorganization. The mobile robot platform can be shared by the three operations. The mobile robot is equipped with different manipulators to complete different tasks. The configuration of the mobile robot and entity model is shown in Figure 1. It is mainly composed of a control box, a double walking arm, a double operation arm, a double walking wheel and its clamping mechanism, and several main parts. The operation arm 1 is fixed on the machine body and it has three DOF which are rotation, stretch, and vertical movement. In addition to the three DOF of the operation arm 1, the operation arm 2 also has a horizontal joint, which can move laterally along the body to realize the pushing out and loading of the insulator string. The dual operation arms have a total of seven three. The double operation arms end effector can be reorganized and installed with different manipulators, 
TABLE 1: Configuration analysis of insulator (auxiliary) replacement robot.

\begin{tabular}{|c|c|c|c|c|}
\hline \multicolumn{5}{|c|}{ Insulator (auxiliary) replacement operation } \\
\hline & \multicolumn{2}{|c|}{ Robot mobile platform } & \multicolumn{2}{|c|}{ Robot operation manipulator } \\
\hline & Operation arm 1 & Operation & Manipulator 1 & Manipulator 2 \\
\hline $\begin{array}{l}\text { Number of } \\
\text { DOF> }\end{array}$ & $\begin{array}{c}\text { Vertical/stretch/rotation (3 } \\
\text { units) }\end{array}$ & $\begin{array}{l}\text { Horizontal/vertical/stretch/ } \\
\text { rotation (4 units) }\end{array}$ & $\begin{array}{l}\text { Clamping/pushing block } \\
\text { mechanism ( } 3 \text { units) }\end{array}$ & Clamping mechanism (1 unit) \\
\hline Function & $\begin{array}{c}\text { Reach operation space } \\
\text { through coordinated joint } \\
\text { movement }\end{array}$ & $\begin{array}{c}\text { Reach operation space } \\
\text { through coordinated joint } \\
\text { movement }\end{array}$ & $\begin{array}{l}\text { The bowl head hanging plate } \\
\text { clamps the W pin and pushes } \\
\text { in }\end{array}$ & $\begin{array}{l}\text { The insulator is clamped and } \\
\text { pushed out/loaded by the stretch } \\
\text { mechanism }\end{array}$ \\
\hline
\end{tabular}

TABLE 2: Configuration analysis of the operation robot for drainage plate bolt tightening.

\begin{tabular}{|c|c|c|c|c|}
\hline \multicolumn{5}{|c|}{ Drainage plate bolt tightening operation } \\
\hline & \multicolumn{2}{|c|}{ Robot mobile platform } & \multicolumn{2}{|c|}{ Robot operation manipulator } \\
\hline & Operation arm 1 & Operation arm 2 & Manipulator 1 & Manipulator 2 \\
\hline $\begin{array}{l}\text { Number } \mathrm{f} \\
\text { DOF }\end{array}$ & Vertical/stretch/rotation (3 units) & $\begin{array}{l}\text { Horizontal/vertical/stretch/ } \\
\text { rotation (4 units) }\end{array}$ & $\begin{array}{l}\text { Bolt fixing mechanism } \\
\text { (0 units) }\end{array}$ & $\begin{array}{l}\text { Bolt tightening } \\
\text { mechanism (1 unit) }\end{array}$ \\
\hline Function & $\begin{array}{l}\text { Reach operation space through } \\
\text { coordinated joint movement }\end{array}$ & $\begin{array}{l}\text { Reach operation space through } \\
\text { coordinated joint movement }\end{array}$ & $\begin{array}{l}\text { Realize the fixation of } \\
\text { the bolt head }\end{array}$ & $\begin{array}{c}\text { Realize nut tightening } \\
\text { operation }\end{array}$ \\
\hline
\end{tabular}

TABLE 3: The configuration analysis of the damper maintenance robot.

\begin{tabular}{|c|c|c|c|c|}
\hline \multicolumn{5}{|c|}{ Damper replacement operation } \\
\hline & \multicolumn{2}{|c|}{ Robot mobile platform } & \multicolumn{2}{|c|}{ Robot mobile platform } \\
\hline & Operation arm 1 & Operation arm 2 & Manipulator 1 & Manipulator 2 \\
\hline $\begin{array}{l}\text { Number of } \\
\text { DOF }\end{array}$ & Vertical/stretch/rotation (3 units) & $\begin{array}{l}\text { Horizontal/vertical/stretch/ } \\
\text { rotation (4 units) }\end{array}$ & $\begin{array}{c}\text { Clamping } \\
\text { mechanism (1 unit) }\end{array}$ & $\begin{array}{l}\text { Pitch/bolt tightening } \\
\text { mechanism ( } 2 \text { units) }\end{array}$ \\
\hline Function & $\begin{array}{l}\text { Reach operation space through } \\
\text { coordinated joint movement }\end{array}$ & $\begin{array}{l}\text { Reach operation space through } \\
\text { coordinated joint movement }\end{array}$ & Clamping damper & $\begin{array}{c}\text { Realize different angle nut } \\
\text { tightening operations }\end{array}$ \\
\hline
\end{tabular}

respectively, to complete the three functions of insulator replacement, drainage plate bolt tightening, and damper replacement.

2.2. The Key Postures of the Robot Operation Process. After the insulator maintenance robot is online, the dual operation arms can be adjusted from the initial posture to the working posture, and they travel along the wire and detect and locate the suspension clamps to achieve rough positioning to the insulator string. By fine-tuning each joint of the operation arm, the positioning of the bowl head hanging plate, $\mathrm{W}$ pin, and insulator steel cap can be completed and the bowl head hanging plate can be clamped, the $\mathrm{W}$ pin can be pushed out, the insulator steel cap can be clamped, and the insulator ball head is pushed out so that the insulator string can be changed from a fixed state to a free state, so as to facilitate manual replacement. The motion simulation of the operation planning entity is shown in Figure 2.
After the drainage board maintenance robot is online, the dual operation arms can be adjusted from the initial posture to the working posture and travel along the wire, detecting and positioning the press-connection-pipe to determine the initial position of the robot to tighten the bolts. The adjustment can be completed by adjusting the joints of the operation arm. The positioning of the bolt head, nut, and the connection with the operation manipulator can be realized. The entity motion simulation of the operation planning is shown in Figure 3.

After the damper robot is online, the dual operation arms can be adjusted from the initial posture to the working posture, travel along the wire, and roughly be positioned to the replacement workspace of the damper, which can be completed by adjusting the joints of the operation arm, especially the movement of the pitch mechanism, the bolt head, nut positioning and the realization of the docking with the operation manipulator and the bolt head and nut, the tightening of the nut, and the clamping of the damper; the 


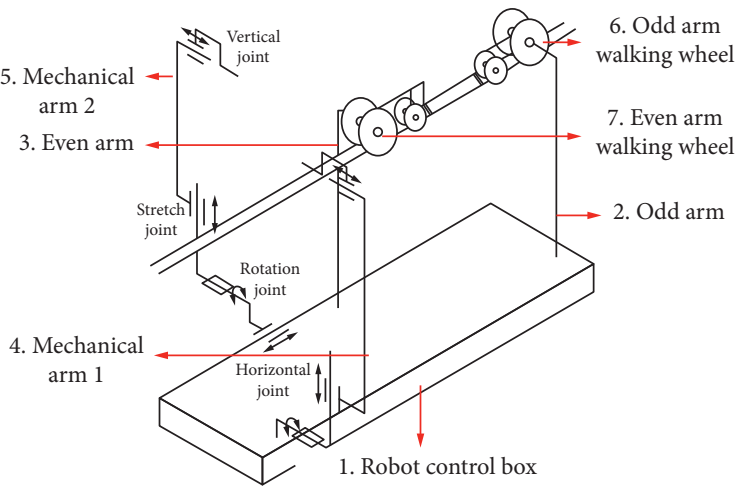

(a)

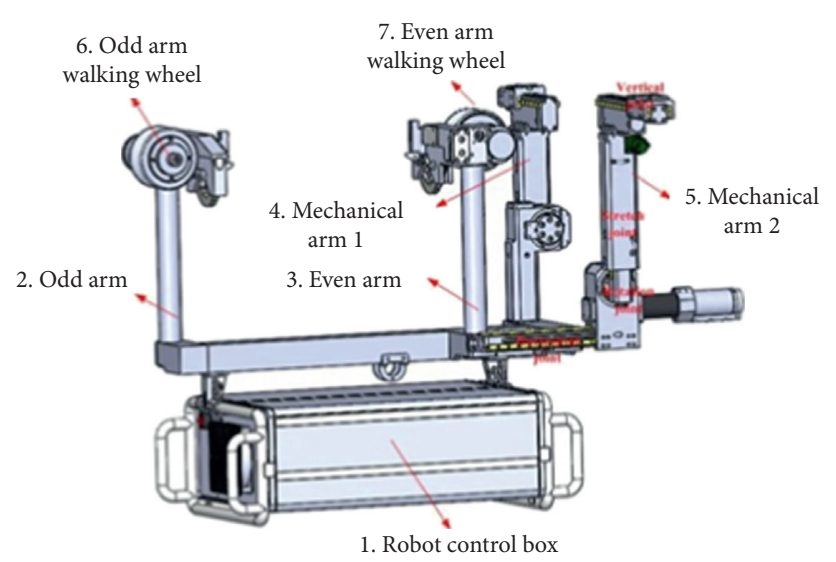

(b)

Figure 1: Configuration and entity model of live maintenance robot mobile platform. (a) Configuration. (b) Entity model.

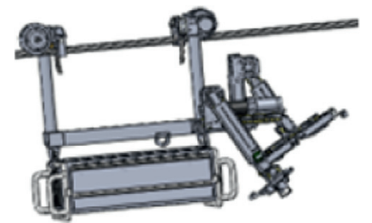

(a)

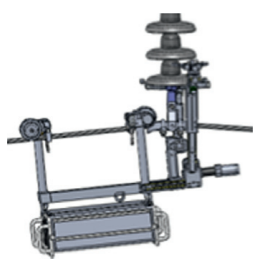

(b)

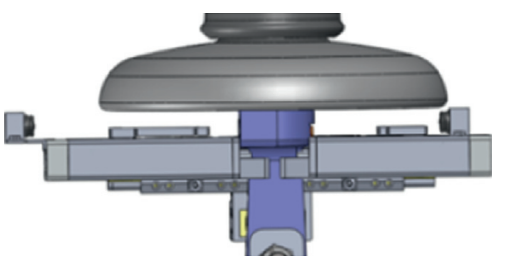

(c)

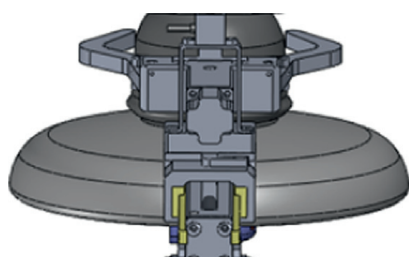

(d)

Figure 2: Insulator replacement operation planning entity motion simulation. (a) Initial posture. (b) Working posture. (c) Bowl head plate clamping. (d) Insulator steel cap clamping.

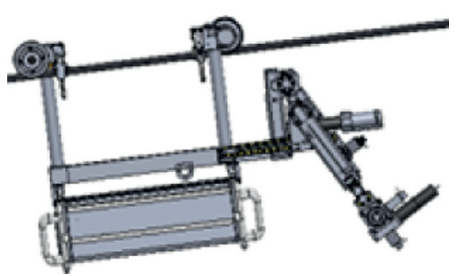

(a)

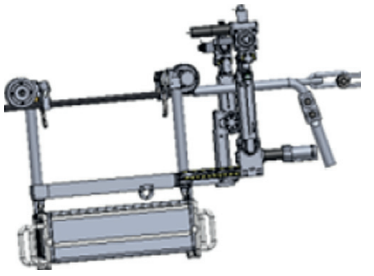

(b)

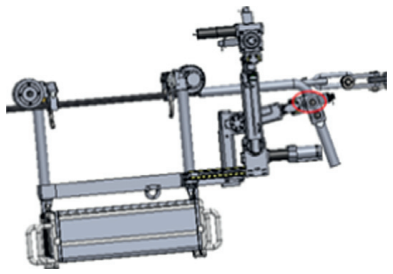

(c)

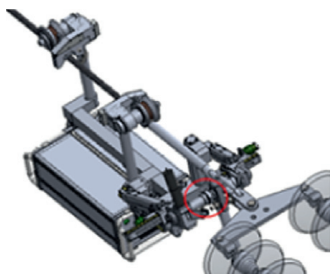

(d)

FIGURE 3: Entity motion simulation of bolt tightening plan for drainage plate. (a) Initial posture. (b) Working posture. (c) Fixing of bolt head. (d) Bolt tightening operation.

physical motion simulation of the operation planning is shown in Figure 4.

\subsection{The Design Principle of Robot Terminal Reconfiguration} for Multitasks. The reconfiguration of the robot operation terminal can be divided into two categories which are static reconfiguration and dynamic reconfiguration. The static reconfiguration is the assembly and reorganization of the terminal by the operator. Dynamically reconfigurable is the movement of the robot's own modules to replace the operation end. Combining with the actual application requirements of special power operation robots, this article adopts the realization method of terminal static reconfiguration. According to the operation task of the live maintenance robot, the corresponding operation end reconstruction principle has been proposed. For different operation tasks, corresponding actuators are required and the structure of the robot is modularized. According to different tasks, the corresponding actuators are selected to reorganize the robot operation ends. According to the functions of each part of the robot, the robot can be divided into two categories. The first category is the operation manipulator, including the bowl head hanging plate clamping and $\mathrm{W}$ pin-pushing end, insulator string pushing end, bolt fixing and bolt tightening end, damper clamping and supporting mechanism, and damper bolt tightening and loosening mechanism. The operation end directly acts on the operation object, making small-scale movements and finetuning the posture as needed. The second category is the 


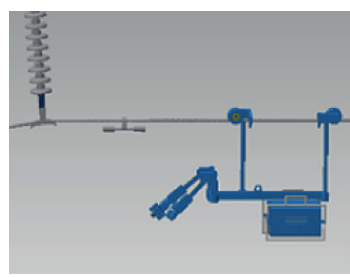

(a)

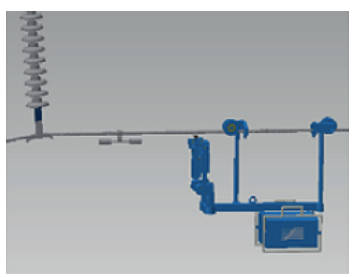

(b)

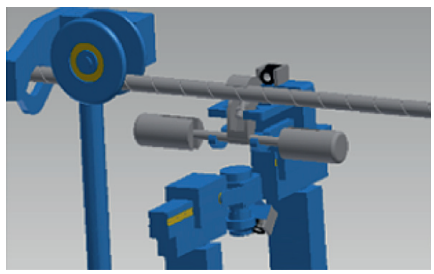

(c)

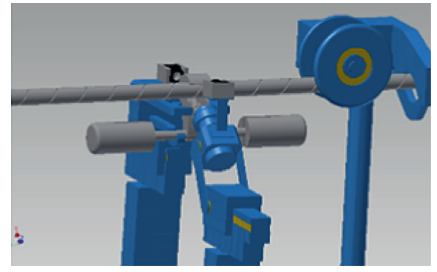

(d)

FIGURE 4: Entity motion simulation of the damper replacement robot. (a) Initial posture. (b) Working posture. (c) Fixing bolt head. (d) Bolt tightening operation.

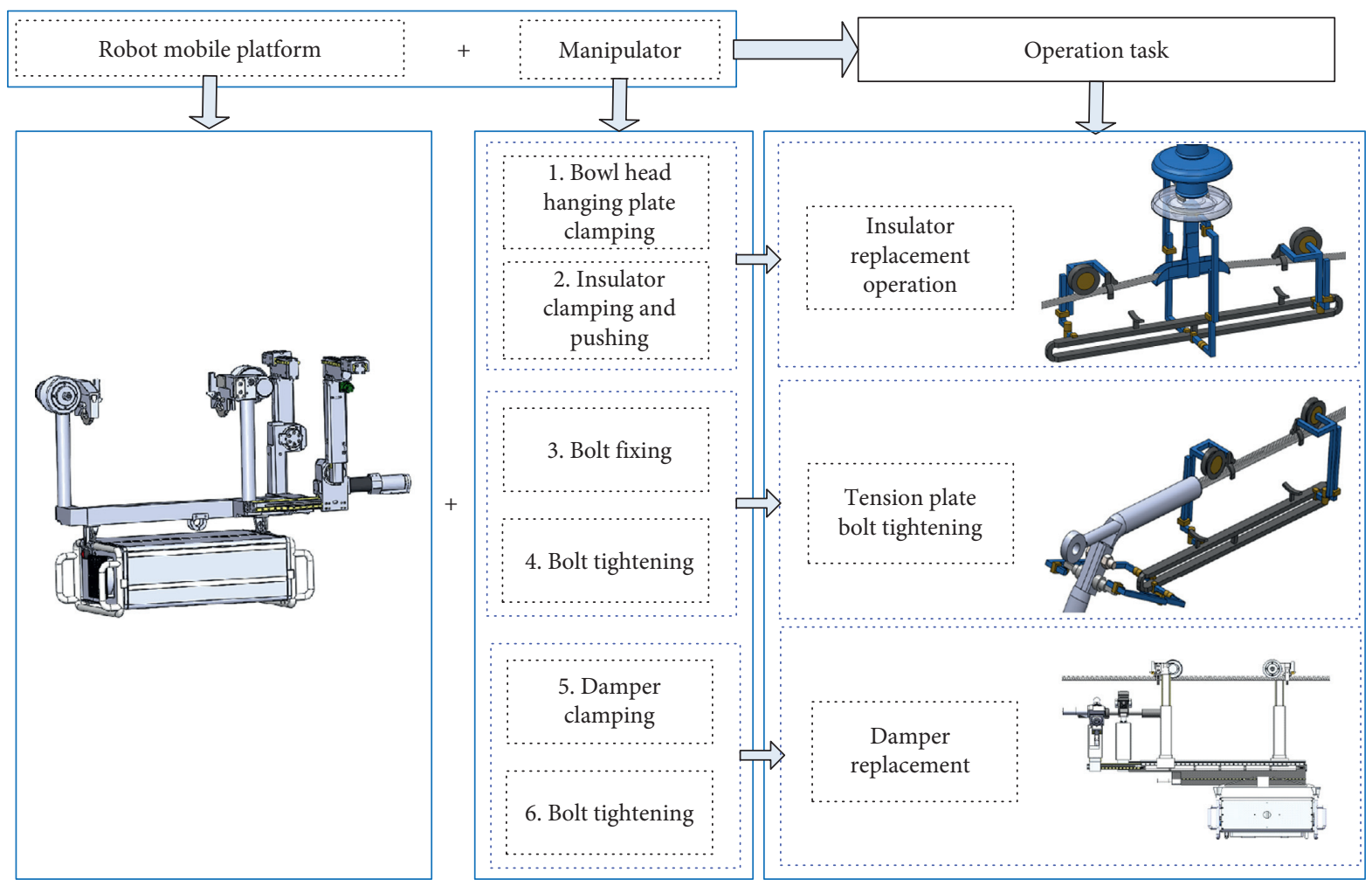

Figure 5: The principle of reconfigurable modular design for multiple tasks.

mobile robot platform for live maintenance, which includes dual manipulators, dual operation arms, and mobile platforms. Each manipulator is composed of multiple joints in series, and each joint moves in coordination to perform terminal positioning or coordinate with the corresponding terminal. The dual mobile arms and dual manipulators coordinate and cooperate to complete different tasks of walking. The mobile platform is the carrier of the entire robot. The mobile platform of the live maintenance robot completes the corresponding operation tasks by carrying different operation ends. The principle of reconfigurable end functions is shown in Figure 5. According to the principle of module division and terminal reconstruction, insulator (auxiliary) replacement, bolt tightening, and damper replacement live working robots are all composed of double operation arms and the mobile robot equipped with double operation manipulators and the mobile robot is used as refactoring the platform. For other multitask robots terminal reconstruction methods can be designed and implemented based on this principle.

\section{Robot Operation Behavior Analysis and Planning Control}

3.1. Analysis of Robot Operation Behavior. In order to clearly describe the robot operation process, the state vector is used to describe the robot key posture. The robot operation can be regarded as the change process of the robot key posture caused by the robot execution. Table 4 shows the definition of the robot state vector.

Using the above representation method, the robot has a total of 2 [12] possible states. Because robots face different tasks and different tasks of robots have specific processes, many postures are not allowed to appear when robots are working. Therefore, through analyzing the robot posture, effective postures can be screened out. As shown in Table 5, 
TABLE 4: Robot state vector.

\begin{tabular}{|c|c|c|c|}
\hline Digit & Status meaning & & Parameter \\
\hline 0 & Vertical mechanism state & & 0: move forward; 1 : move backward \\
\hline 1 & Horizontal mechanism state & & 0 : move in; 1 : move out \\
\hline 2 & Stretch mechanism state & Robot mobile platform & 0 : extend; 1 : shorten \\
\hline 3 & Rotation mechanism state & & 0 : rotate clockwise; 1 : rotate counterclockwise \\
\hline 4 & Walking mechanism & & 0: walking; 1 : stop \\
\hline 5 & Insulator clamping mechanism state & & 0 : clamping; 1 : release \\
\hline 6 & Bowl head hanging plate clamping mechanism state & Insulator maintenance & 0 : clamping; 1 : release \\
\hline 7 & $\mathrm{~W}$ pin push mechanism status & & 0 : push out; 1 : push in \\
\hline 8 & Drainage plate bolt tightening mechanism state & Drainage plate maintenance & 0 : tightening; 1 : loosen \\
\hline 9 & Damper clamping mechanism state & & 0 : clamping; 1 : release \\
\hline 10 & Damper bolt tightening mechanism & Damper maintenance & 0 : clamping; 1 : release \\
\hline 11 & Damper bolt tightening pitch mechanism & & 0: up; 1: down \\
\hline
\end{tabular}

TABLE 5: The robot's effective state vector value.

\begin{tabular}{lcc}
\hline State vector & Meaning & Vector value \\
\hline$S_{0}$ & Initial state & $0 \times 0000$ \\
$S_{1}$ & Insulator steel cap clamping state & $0 \times 0001$ \\
$S_{2}$ & Clamping state of bowl head hanging plate & $0 \times 0010$ \\
$S_{3}$ & W pin rolling out status & $0 \times 0011$ \\
$S_{4}$ & Alignment of drainage plate bolts & $0 \times 0100$ \\
$S_{5}$ & Alignment of drainage plate nut \\
$S_{6}$ & Damper clamping state & $0 \times 0101$ \\
$S_{7}$ & Damper bolt alignment state & $0 \times 0110$ \\
$S_{8}$ & Damper clamping mechanism state & $0 \times 0111$ \\
$S_{9}$ & Damper screw bolt mechanism & $0 \times 1000$ \\
$S_{10}$ & Damper bolt tightening pitch mechanism & $0 \times 1001$ \\
$S_{11}$ & Standby state-1 & $0 \times 1010$ \\
$S_{12}$ & Standby state-2 & $0 \times 1011$ \\
$S_{13}$ & Standby state-3 & $0 \times 1100$ \\
$S_{14}$ & Standby state- 4 & $0 \times 1101$ \\
$S_{15}$ & Standby state-5 & $0 \times 1110$ \\
\hline
\end{tabular}

effective state screening can greatly optimize the robot operation flow.

\subsection{Robot Autonomous Operation Behavior Planning}

3.2.1. Definition of Robot Behavior. The robot motion behavior in the operation process from part to the whole mainly includes joint motion behavior, arm motion behavior, robot motion behavior, and robot abnormal behavior processing. Among them, the basic behavior of the robot joint motion behavior (JMB) is defined as a module that is directly connected to the drive mechanism and sensors and has specific motion functions. Manipulator action behavior AMB (arm motion behavior) is a robot arm behavior that can achieve specific functions, which is composed of several basic joint behaviors and a joint behavior inference device as shown in the following equation, where $\mathrm{AMB} i$ is the combination behavior and $\boldsymbol{F}$ is the joint state transition function:

$$
A M P=\left(\mathrm{JMP}_{1}, \mathrm{JMP}_{2} \mathrm{JMP}_{3}, \ldots, \mathrm{JMP}_{n}, F\right) .
$$

Robot motion behavior (RMB) is composed of several operation arm behaviors, as well as the robot walking mechanism and behavior inference device. Its activation and execution are consistent with the operation arm motion behavior. The robot motion behavior is defined as equation (2), wherein AMB is defined in the above and $F$ is the state transition function. The $\mathrm{C}++$ thread pseudocode is used and can be formally expressed as Algorithm 1:

$$
R M P=\left(\mathrm{AMP}_{1}, \mathrm{AMP}_{2} \mathrm{AMP}_{3}, \ldots, \mathrm{AMP}_{n} \text {, Walking Action, } F\right),
$$

Due to the complex working environment of electric power robots, there are many steps in the operation process and strict sequence of steps. In addition, the robot will inevitably encounter some special situations, such as interruption of information transmission, sensor, and industrial computer crash. Therefore, it is necessary to introduce an exception handling mechanism to ensure personnel safety, line safety, equipment safety, and normal operation tasks.

3.2.2. Robot Operation Behavior Planning Based on Task Decomposition. Regarding the specific electric maintenance robot, the whole robot operation process can be divided into four basic behaviors, namely, the behavior of the robot arm 1 , the behavior of the robot arm 2, the behavior of the work 


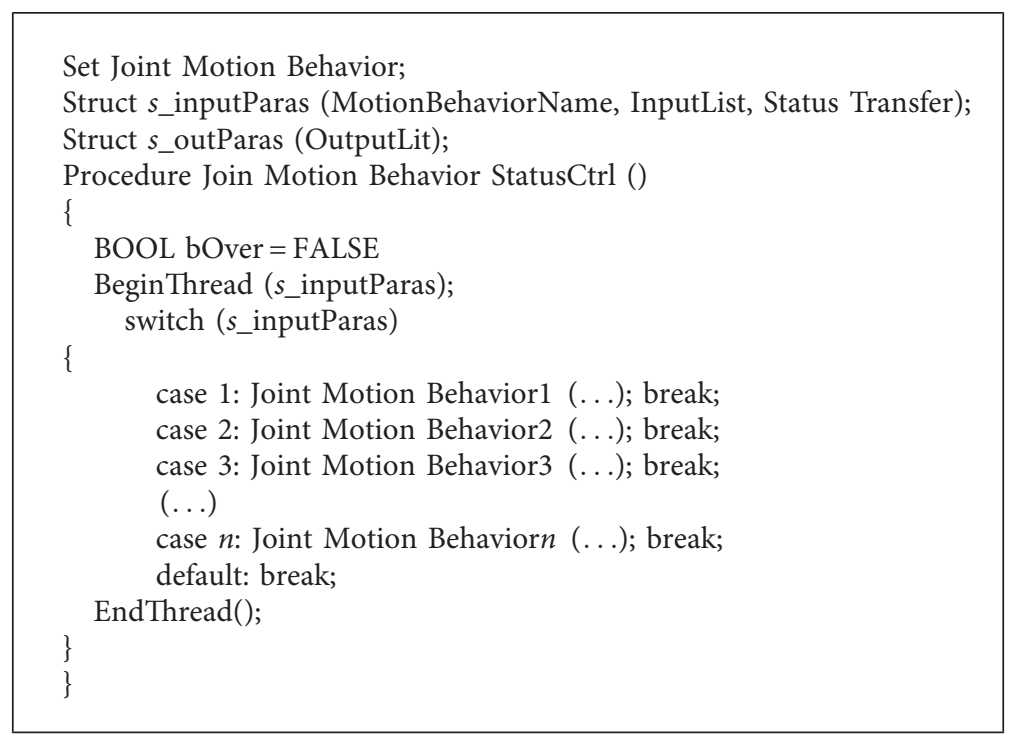

Algorithm 1: Autonomous planning algorithm for robot motion behavior.

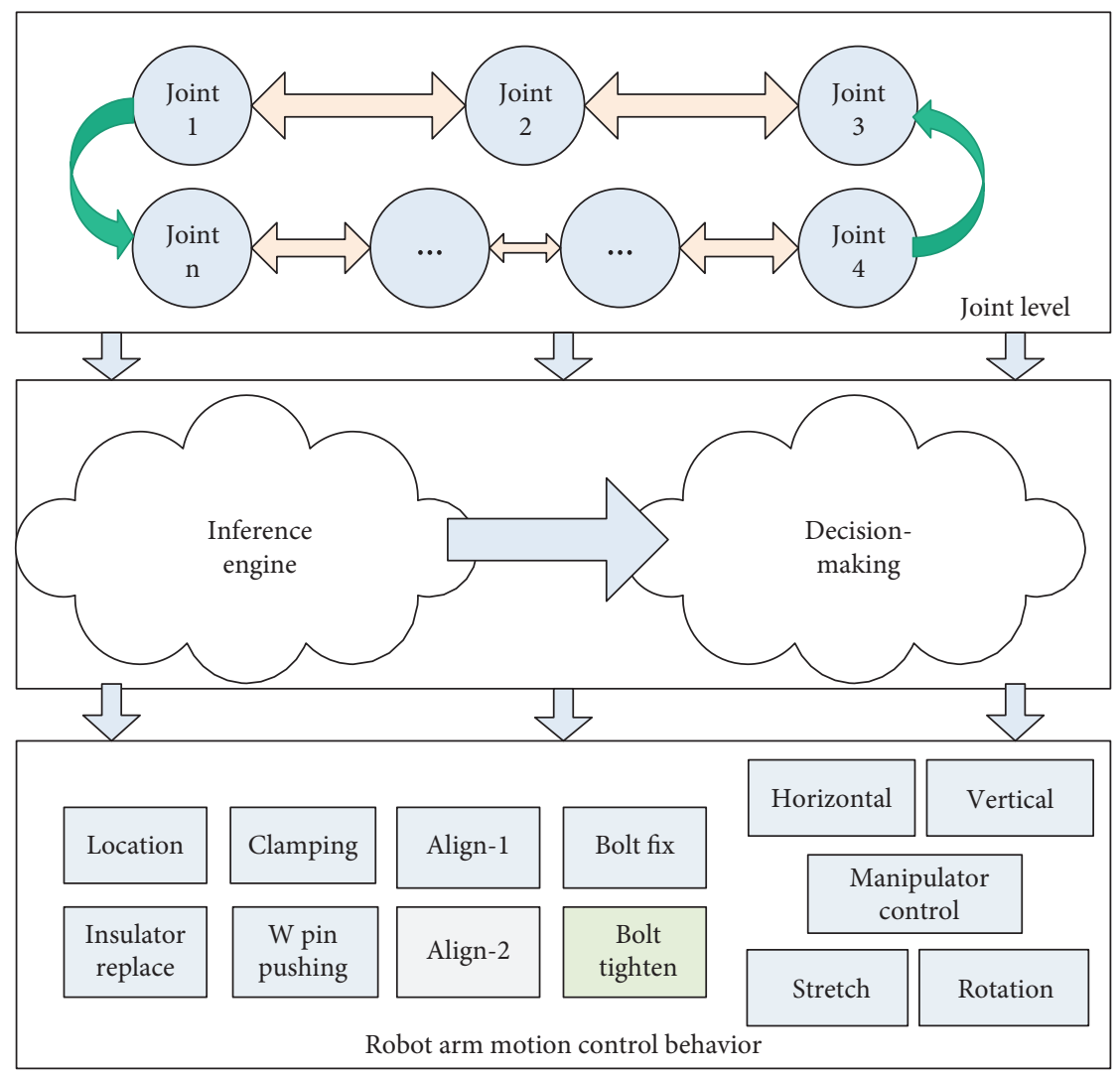

FIgURE 6: Robot task decomposition and behavior planning.

end 1 , and the behavior of the work end 2. Each behavior can be completed by several robot posture adjustments and also includes multiple robot state transitions. For each behavior of the robot, the corresponding state transfer function can be set separately, by inputting the initial state and target state of the robot and behavior parameters to complete the robot state transition. Therefore, the whole process of completing different tasks on the power transmission line by the robot represented by the basic behavior and combined behavior of the robot can be obtained as shown in Figure 6 .

3.2.3. Robot Operation Behavior Control. The architecture of robot autonomous behavior control can be divided into four 


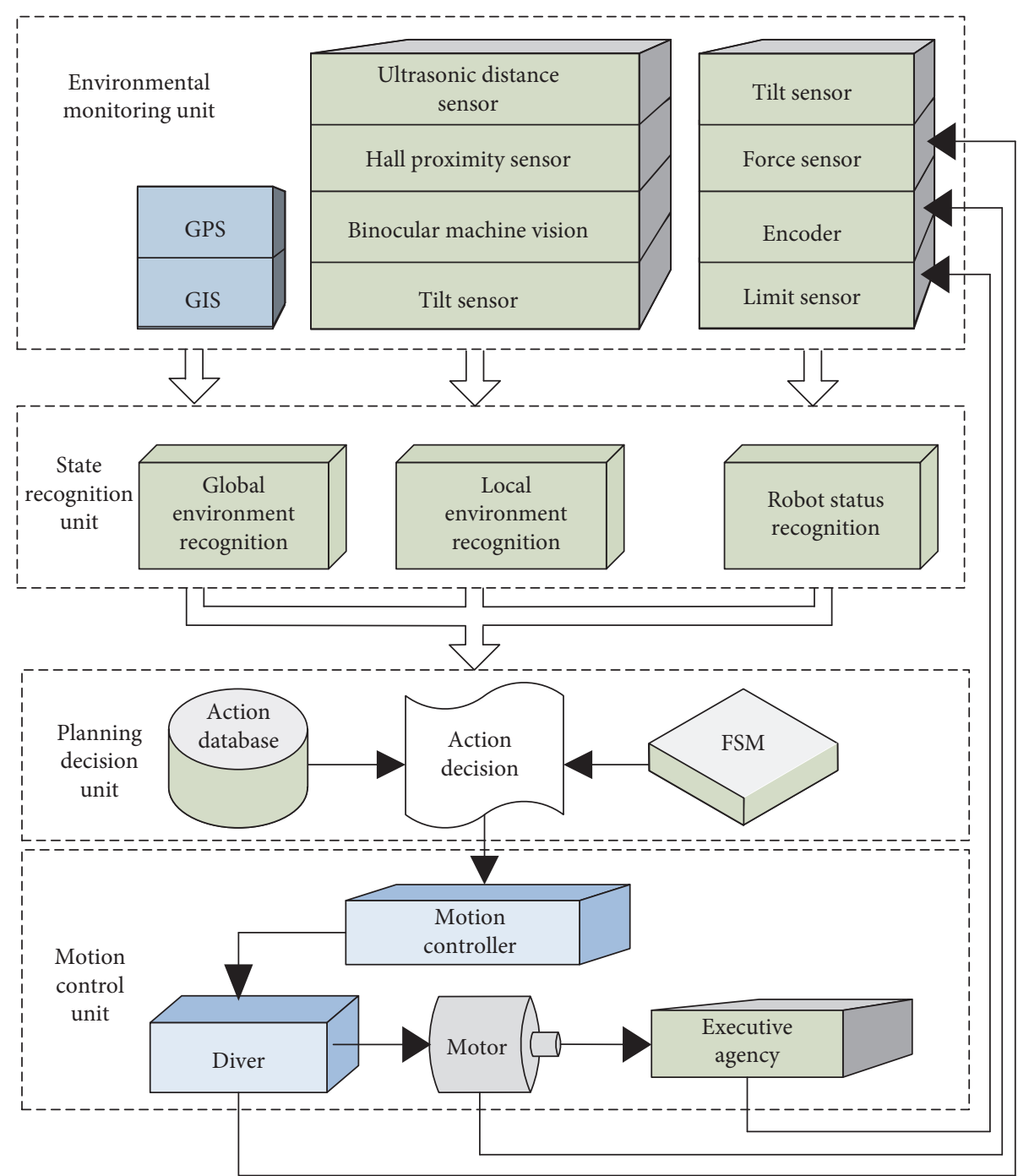

FIGURE 7: Robot schematic diagram of autonomous behavior control.

units as shown in Figure 7, namely, environmental monitoring unit, state recognition unit, planning decision unit, and motion control unit. Among them, the environment monitoring unit is mainly used by the robot to detect and perceive the robot operation environment with various sensors carried by itself, which is also the basis for the robot intelligent decision-making. Based on the monitoring of the robot's local and global environment, the robot can recognize and detect its own operation status. Then, the robot motion planning decision can be made through the behavior database and FSM model, and finally, the motion control unit drives the robot joint motors to realize intelligent autonomous operation control.

\section{Robot Operation FSM and Autonomous Control System Design}

4.1. FSM Design for Insulator Replacement Operation. Finite state machine is a mathematical model that represents a finite number of states and behaviors such as transitions and actions between these states. A finite state machine $M$ is a five-tuple, $M=(K, E, T, S, Z)$ wherein $K$ is a finite set and each element in it is called a state. $E$ is a finite alphabet and each element of it is called an input character. $T$ is a conversion function which is a mapping on $K \times E \longrightarrow K$. $S$ is an element in $K$, which is the only initial state. $Z$ is a subset of $K$, a final state set. When the online maintenance robot is performing insulator replacement operations, the robot dual operation arms and their ends need to take a series action, including multiple states and state transition rules. These actions have been proved to be the most feasible way through many experiments. They are set according to the operation object task and the robot's own mechanism. It is also based on experience planning. The robot FSM design for insulator replacement is shown in Figure 8. The operation can be divided into nineteen states, which are triggered by nineteen events. The event can be described as follows: (1) operation arm 1 turns forward; (2) operation arm 2 turns forward; (3) robot goes online; (4) operation arm 1 turns backward; (5) operation arm 2 turns backward; (6) operation arm 2 extends; (7) the robot moves forward at a low speed; (8) the robot walking wheel touches the suspension clamp; 


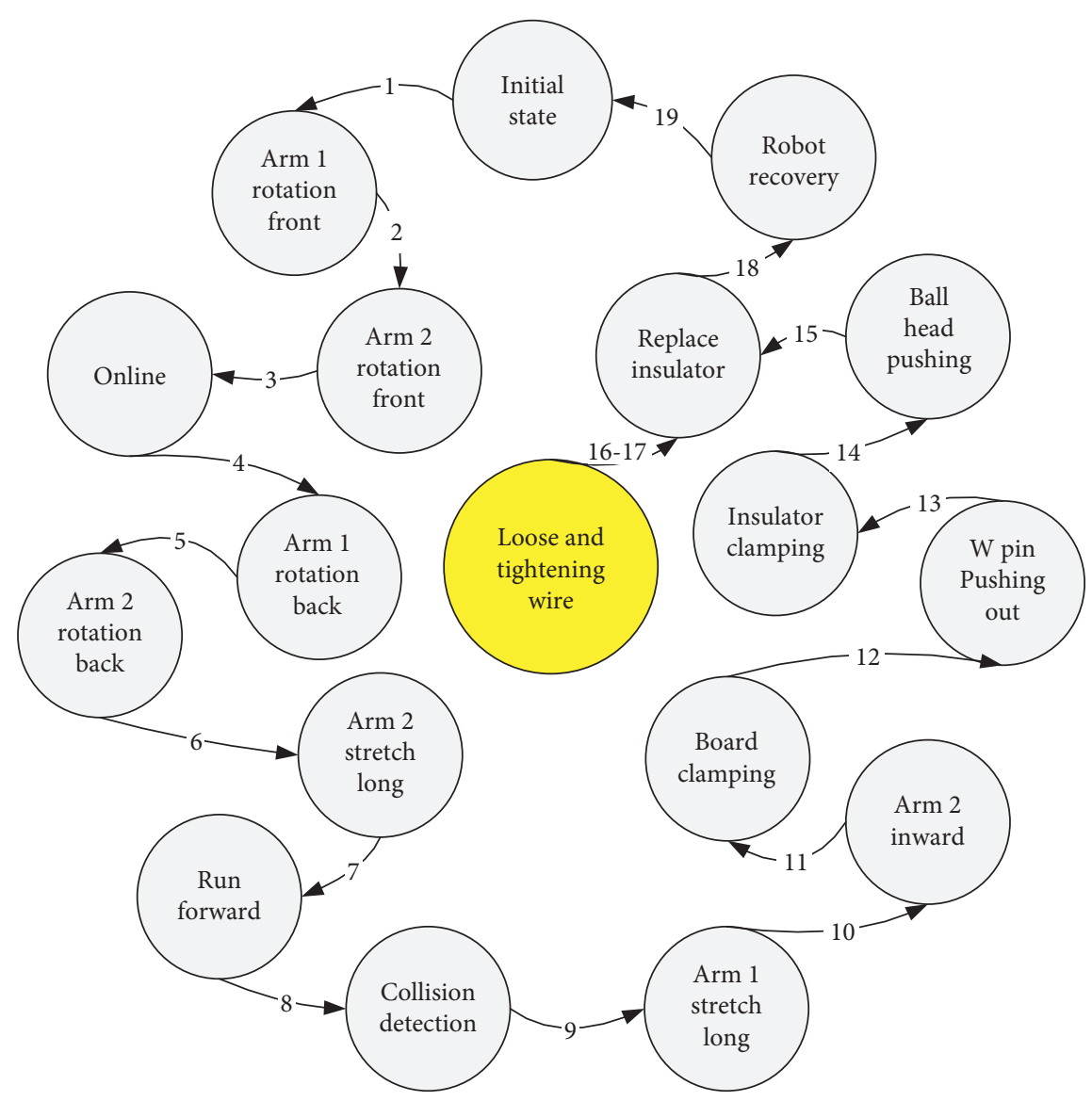

FIGURE 8: FSM design for insulator replacement operation.

(9) the operation arm 1 extends; (10) the operation arm 1 moves inward; (11) the operation hand 1 clamps the bowl head hanging plate; (12) push out W pin; (13) clamping of the insulator; (14) push out the ball head; (15) replace the insulator; (16) loose the wire; (17) tightening the wire; (18) restore the robot posture; and (19) return the robot to the initial state.

4.2. FSM Design for Drainage Plate Bolt Tightening Operation. According to the abovementioned bolt tightening operation motion planning and manual experience operation, the FSM design when the robot performs the drainage plate tightening operation can be obtained as shown in Figure 9. The operation can be divided into eighteen states, which are triggered by eighteen events. The event description is as follows: (1) operation arm 1 turns forward; (2) operation arm 2 turns forward; (3) robot goes online; (4) operation arm 1 turns backward; (5) operation arm 2 turns backward; (6) operation arm 2 extends; (7) the robot moves forward at a low speed; (8) the robot walking wheels collide with the press-connection-pipe; (9) operation arm 2 moves forward; (10) operation arm 1 turns forward; (11) operation manipulator 2 moves forward; (12) both arms move inward; (13) operation manipulator 1 fixes the bolt head; (14) operation manipulator 2 tightens the nut; (15) the nut has been tightened and the operation is completed; (16) the operation end exited; (17) and the robot returns to the initial state.

4.3. FSM Design for Damper Replacement Operation. According to the motion planning of the damper operation and manual experience operation, the FSM design of the robot during the replacement of the damper can be obtained as shown in Figure 10. The operation can be divided into nineteen states, which are triggered by nineteen events. The event description is as follows: (1) operation arm 1 turns forward; (2) operation arm 2 turns forward; (3) robot goes online; (3) operation arm 2 turns backward; (4) robot coarse positioning damper; (5) operation arm 1 extends; (6) operation arm 1 moves inward; (7) operation arm 2 extends; (8) operation arm 2 pitch; (9) bolt align; (10) align success; (11) align fail; (12) fix bolt head success; (13) fix bolt head fail; (14) arm 1 stretch; (15) arm 1 stretch inward; (16) manipulator 2 tightens the nut; (17) operation is completed; (18) operation end exited; and (19) the robot returns to the initial state.

4.4. Design of Object-Oriented Autonomous Operation Control System. Generally speaking, the robot operation process can be divided into two behaviors which are arm motion and 


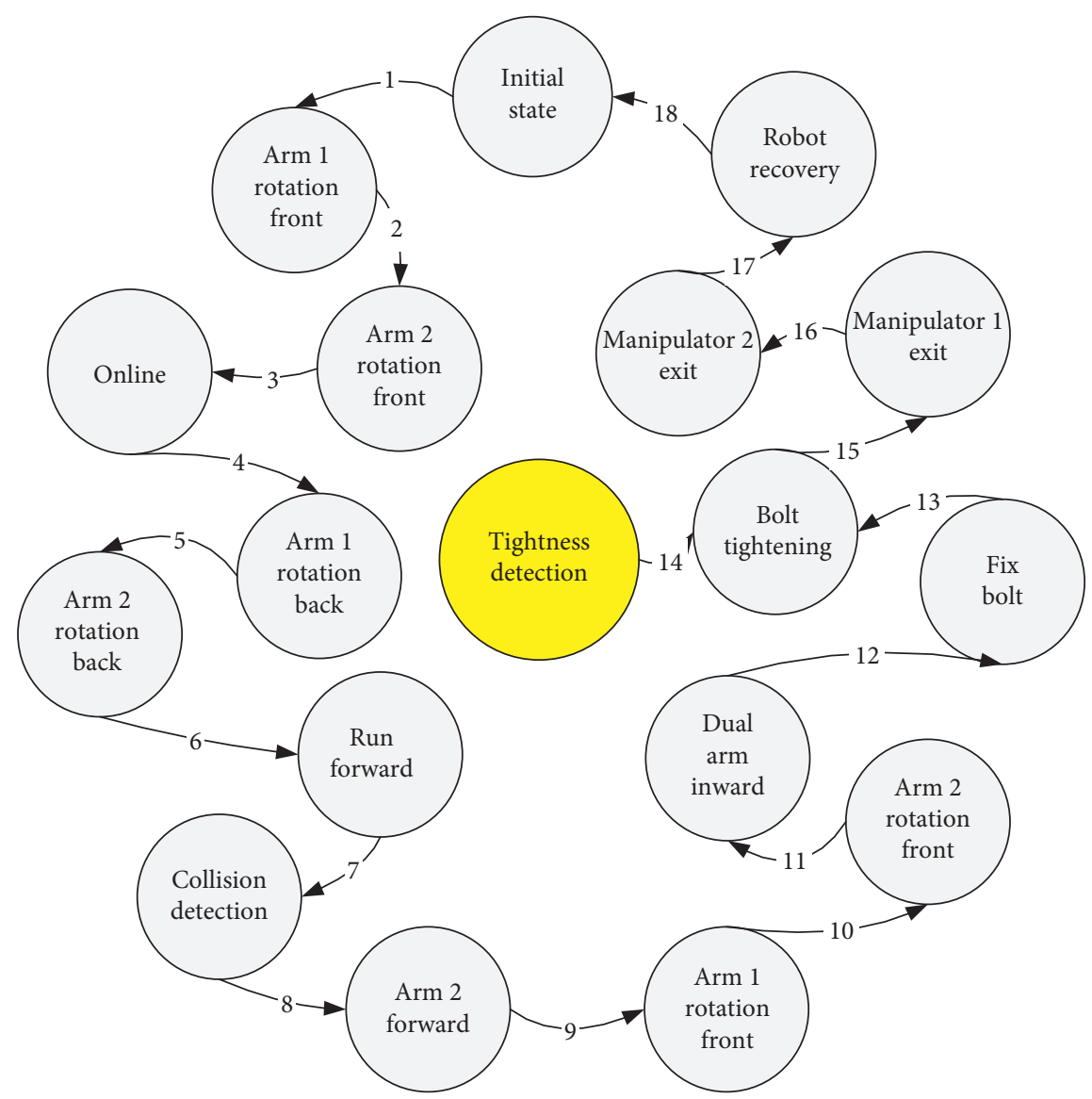

FIGURE 9: FSM design of drainage plate tightening operation.

end-effector motion. Each behavior includes detailed steps for executing each motor. The robot compiles the robot operation behavior plan according to the task type and stores it in the robot database. The robot autonomously selects the behavior plan to execute the action after detecting the operation object; that is, the robot behavior planning sequence is established as shown in Figure 11. The sequence includes the motor steps and parameters from the robot detection of the operation object to the end effector. The robot behavior planning sequence is the optimal motion plan set by the designer based on the robot experience value. During the robot autonomous operation, the behavior will be interrupted due to some uncontrollable factors such as the PC restart. Therefore, the robot is required to have the ability to self-recover behavior after the system restarts. The premise is that the robot can "memorize" the motor running value and the corresponding key sensor information in the motion sequence step executed before the failure after the industrial computer restarts; then the robot can use this to continue the motion plan at the breakpoint. Therefore, the state parameter sequence is added on the basis of the robot behavior motion planning sequence and stored in the robot database system to store the robot real-time status information, including the number of steps in the running action sequence, the execution status of this step, the hall sensor light potential information, count value of the motor executed in this step, and tilt sensor information. The step in the state parameter sequence corresponds to the steps in the robot action sequence. The status contains three states "not started," "end," and "in progress." The hall sensor records information about each actuator's collision or in position. The motor count value can record the motor's running mileage in the current step in real time. The inclination sensor information reflects the robot's posture state at this time, and its autonomous operation control can be realized through the interaction of sensor information carried by the robot itself.

After the robot restarts from the breakpoint, it first recognizes the step and status in the state parameter sequence and then combines the motor count value and sensor information value to complete the continuation of the action planning sequence. In the process of robot adaptive operation, the state information sequence can save the movement and environment information under the current behavior of the robot in real time and provide detection and decisionmaking functions for the robot's autonomous operation behavior. The flowchart of the robot autonomous operation principle is shown in Figure 12. The object-oriented design 


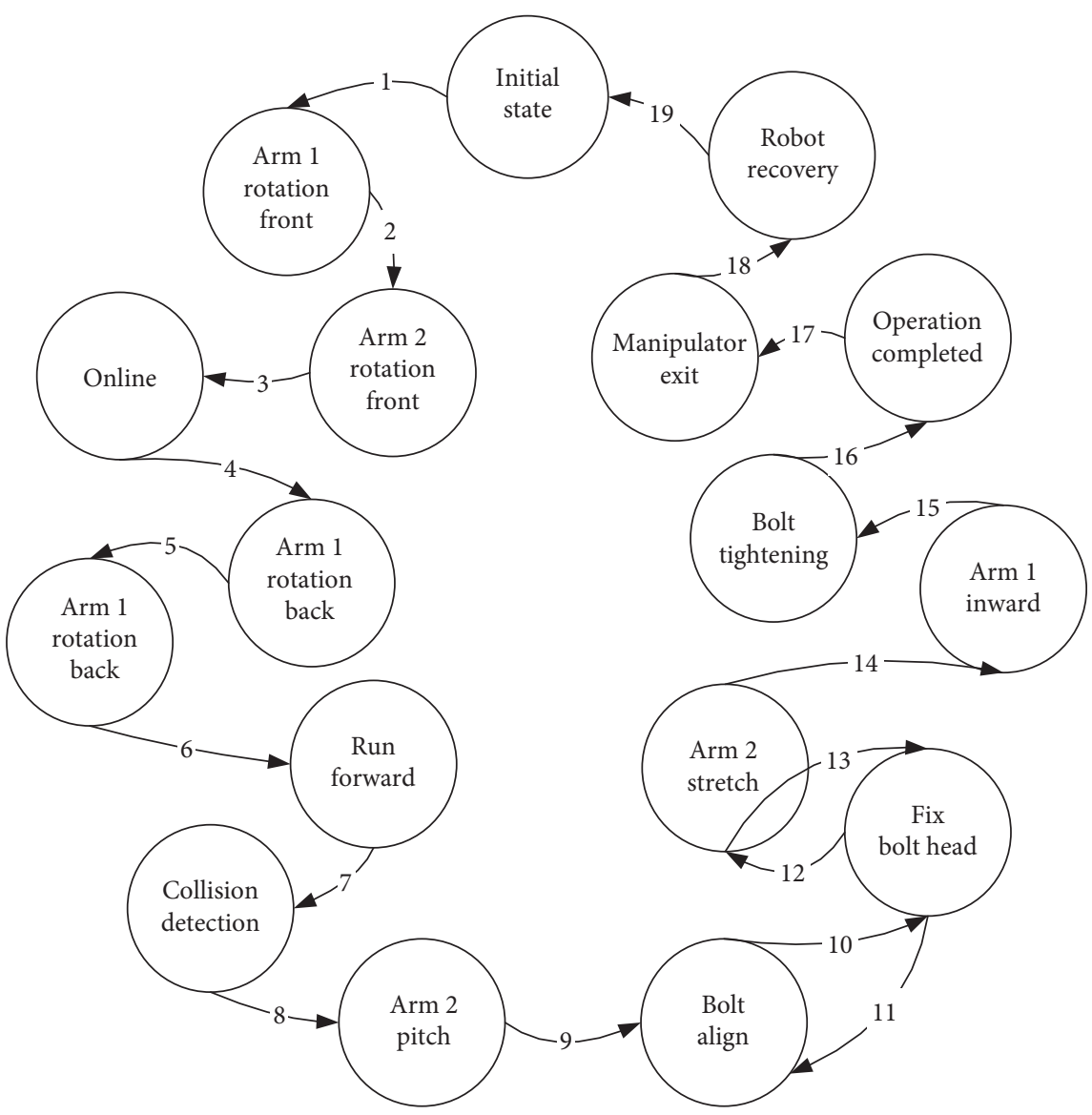

FIGURE 10: FSM design for damper replacement robot.

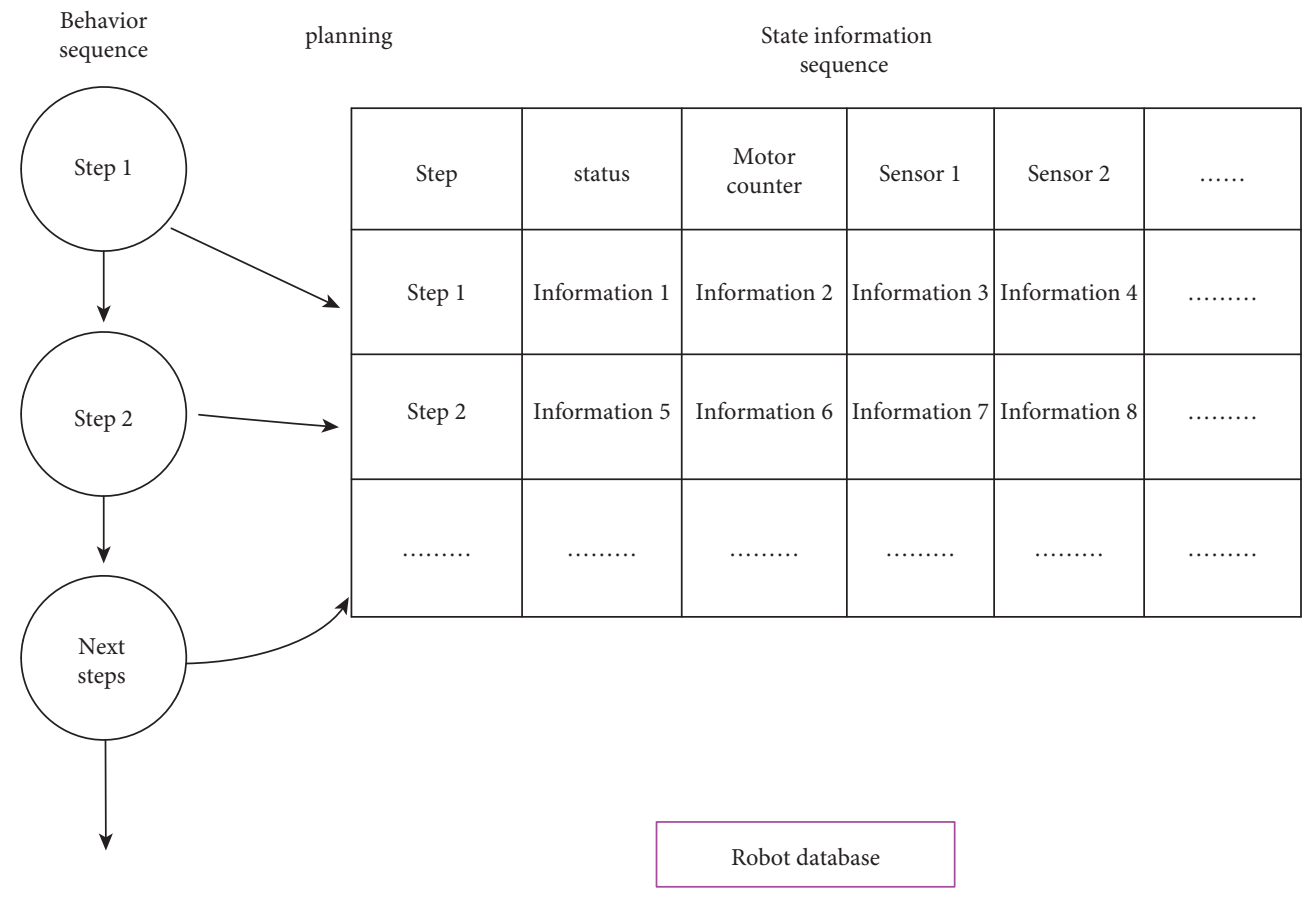

FIGURE 11: Robot status information sequence. 


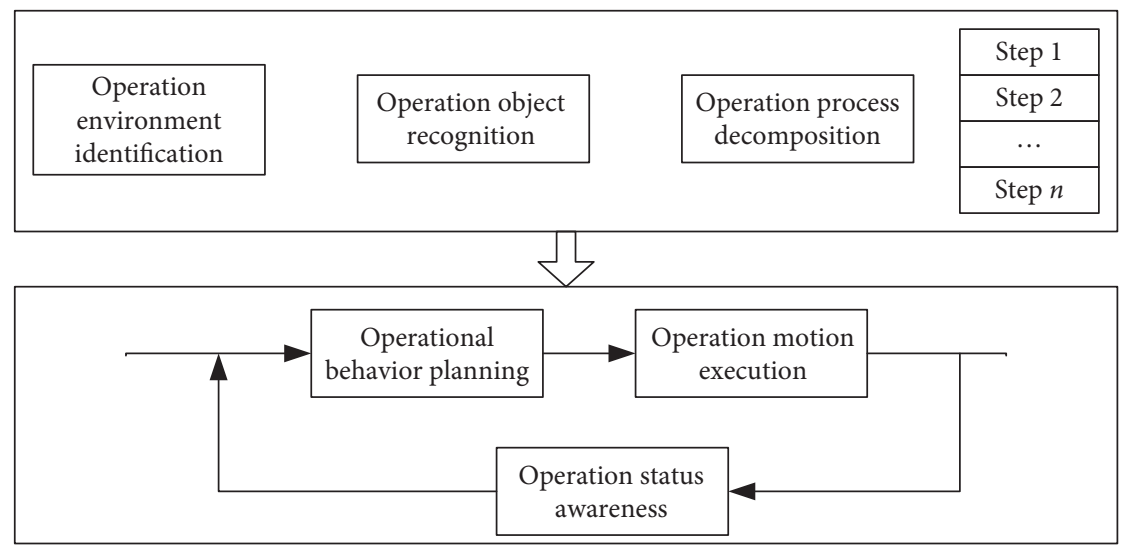

FIgURE 12: Schematic diagram of robot autonomous operation.

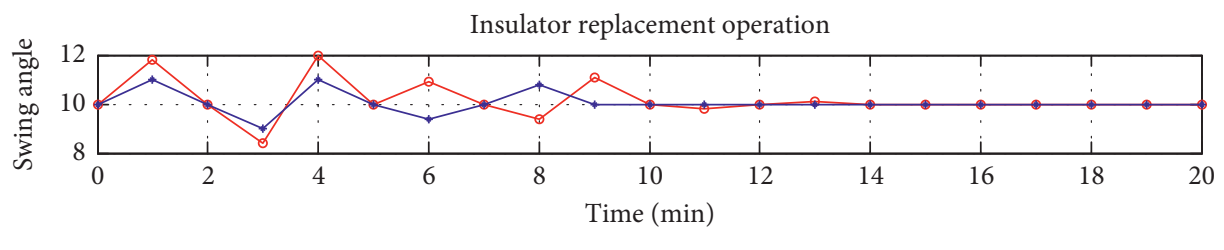

- Traditional control

$\rightarrow$ FSM control

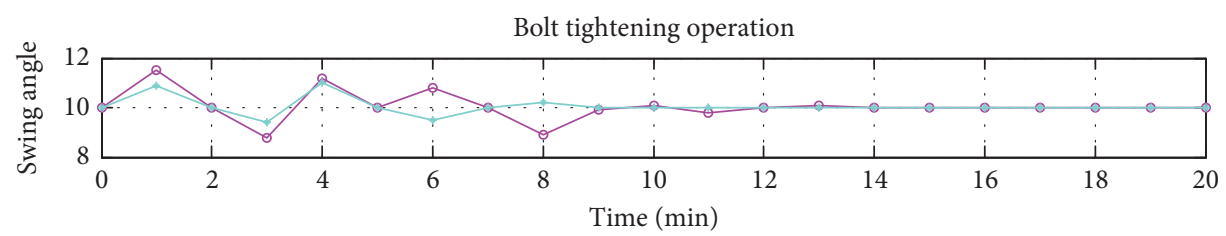

- Traditional control

$\rightarrow$ FSM control

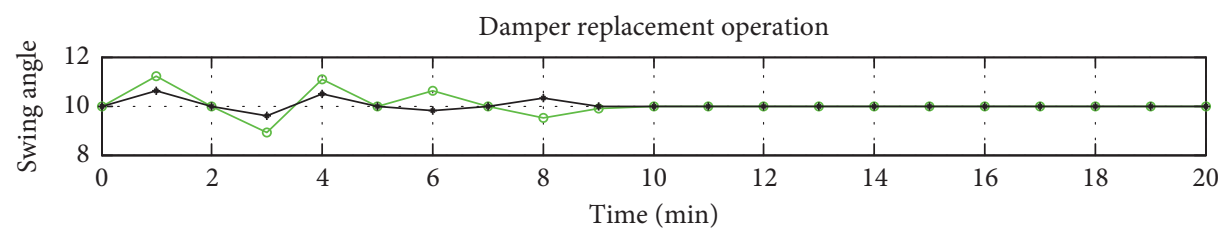

$\rightarrow$ Traditional control

$\rightarrow$ FSM control

FIGURE 13: Robot performance under different methods.

method is used when designing the autonomous operation control system. Compared with the original process-oriented fixed program, the object-oriented design method converts the robot motor control into a natural language description of the robot motion. The realization process of the program is brief and clear. The robot operation steps can be described as the movement of each mechanism, so as to get rid of the attention of the underlying motor movement and the sensor configuration, avoiding the complicated and tedious process of process-oriented design methods. At the same time, the robot operation program design is clear, and the human-machine interaction is simple, which is conducive to the promotion and use of robots subsequent maintenance.

\section{Experimental Research}

5.1. Simulation Experiment. In order to verify the effectiveness of the robot FSM behavior control method, three different tasks have been used as the research objects. For each task, traditional manual tasks and the FSM autonomous behavior tasks proposed in this paper are used to control the robots. The sensor signal carried by itself monitors the working status of the robot in real time to evaluate the robot 


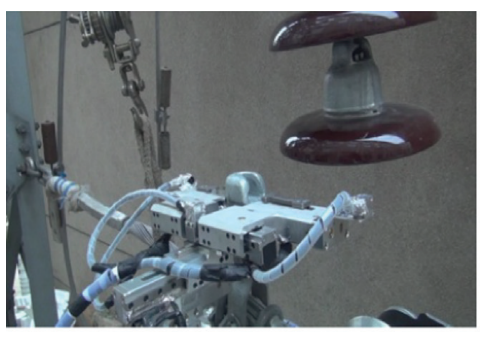

S1: free state

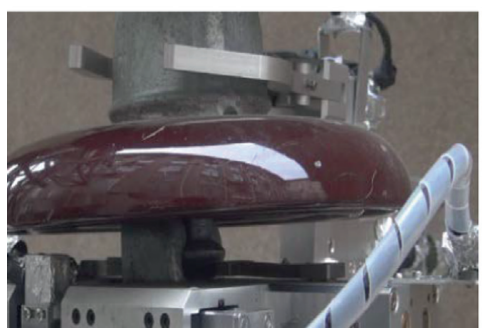

S4: push out W pin

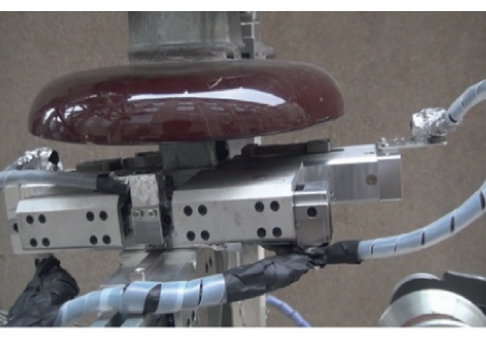

S2: bowl head clamping

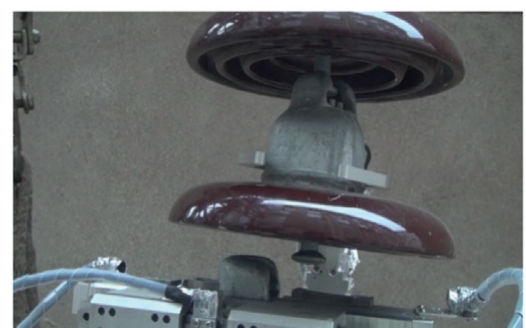

S5: push ball head

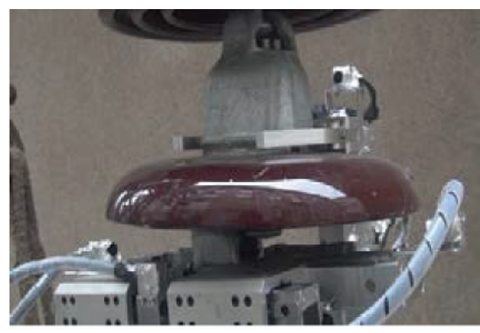

S3: steel cap clamping

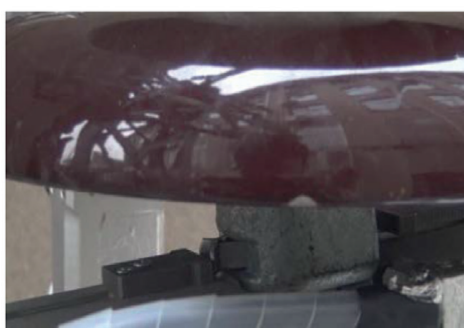

S6: push in $\mathrm{W}$ pin

(a)

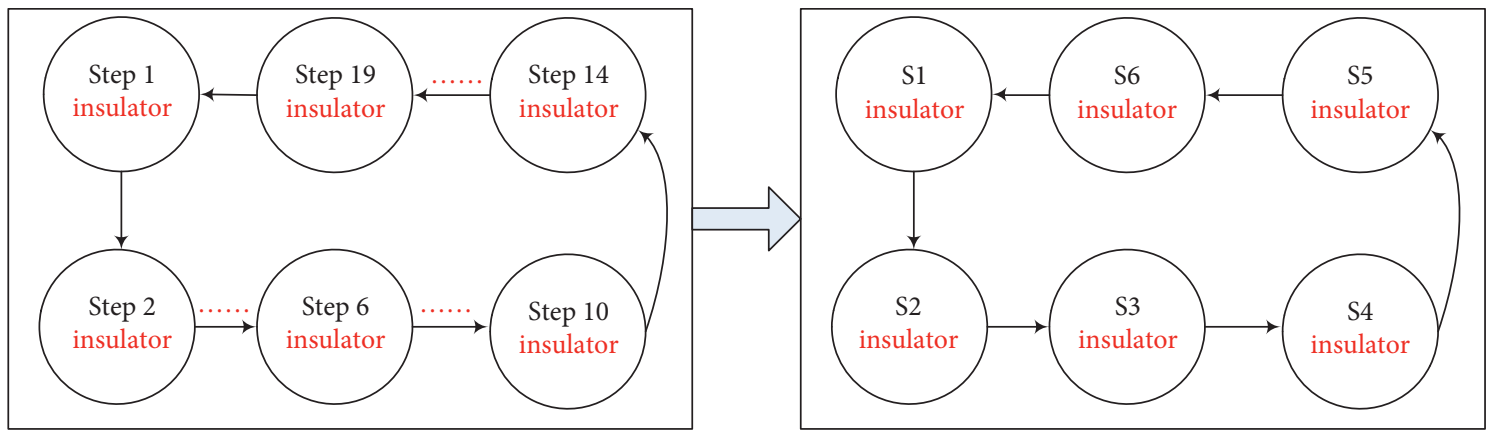

(b)

FIGURE 14: Field experiment of autonomous behavior control for insulator maintenance robot. (a) Insulator operation main process. (b) Insulator operation FSM optimization.

performance under different control methods. The obtained simulation results are shown in Figure 13. The horizontal axis of the three sets of graphs is time and the vertical axis is the robot swing angle. The inclination angle of the robot operation line is 10 degrees and three different operating experiments are performed twice.

Through the three sets of simulation results for insulator replacement, bolt tightening, and damper replacement, it can be seen that, based on the robot operation motion control under the FSM autonomous behavior control method, the system swing angle is smaller than the manual control method and the time for the robot to reach the equilibrium state is less than the manual method, especially in the process of damper replacement being more obvious. The main reason is that under the FSM autonomous behavior control method, robot motion planning has been further optimized. Some redundant motions in the operation process are eliminated. At the same time, the robot multijoint linkage control is integrated in some time periods. Therefore, the robot robustness motion and the robot operation efficiency have been significantly improved. Therefore, the simulation experiment verifies the effectiveness of the FSM-based robot autonomous behavior control method.

5.2. Field Operation Experiment. In order to verify the effectiveness of the robot autonomous behavior control method under different tasks, this paper developed a robot physical prototype system for insulators, drainage plates, and damper maintenance operations, under the jurisdiction of the State Grid Hunan Electric Power Transmission Maintenance Co., Ltd.; three types of operations tests were carried out on the live line. It can be seen from Figure 14 that in the process of insulator replacement using the autonomous behavior control method, the free state insulator-bowl head hanging plate, clamping-steel cap, $\mathrm{W}$ pin-pushing, ball head-pushing, a series of smooth states and behavior transition, and the robot insulator replacement operation can be successfully completed. Due to the intelligent behavior control, the actual operation steps of insulator replacement have reduced from nineteen steps of FSM theoretical model to six; according to the average value of the statistical experiment data, the robot operation 


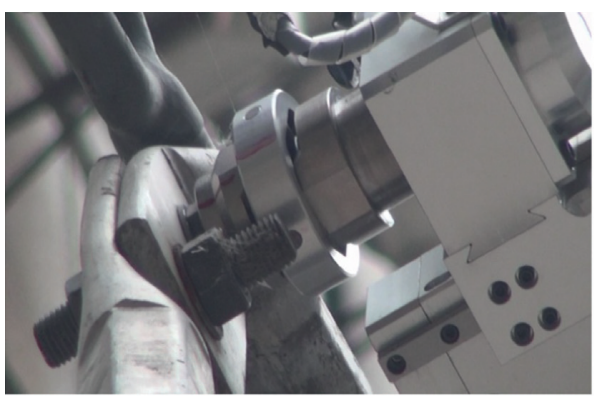

$\mathrm{S}_{1}$ : nut alignment

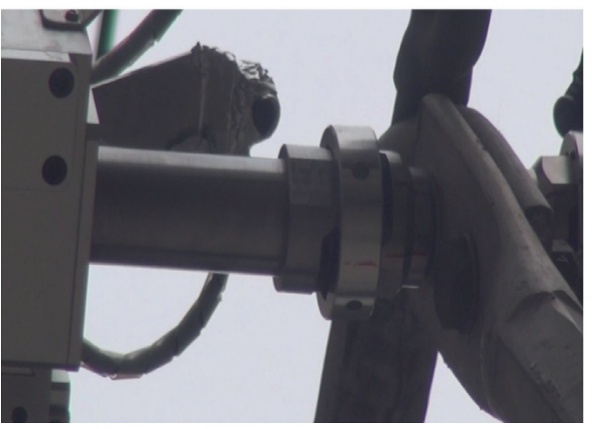

$\mathrm{S}_{3}$ : tightening the nut

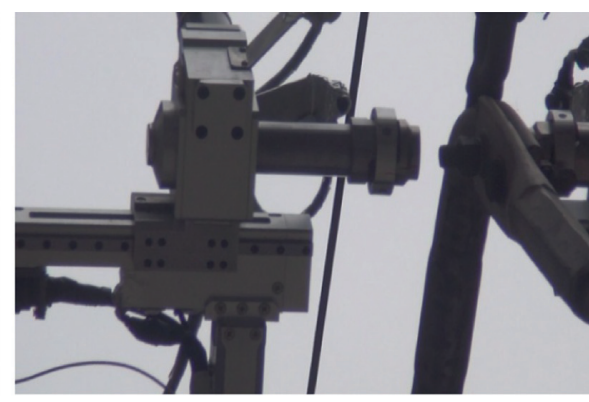

$\mathrm{S}_{2}$ : bolt head alignment

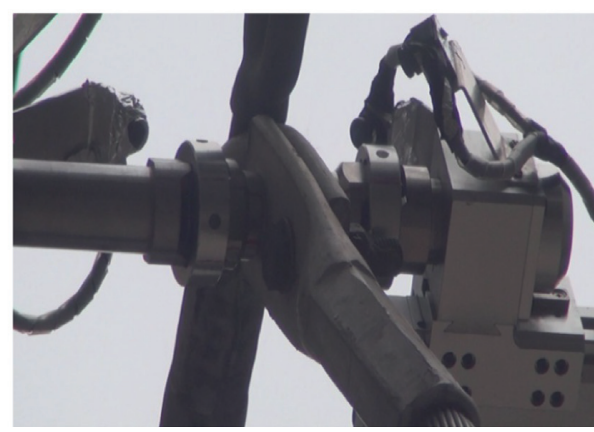

$\mathrm{S}_{4}$ : fixed bolt head

(a)

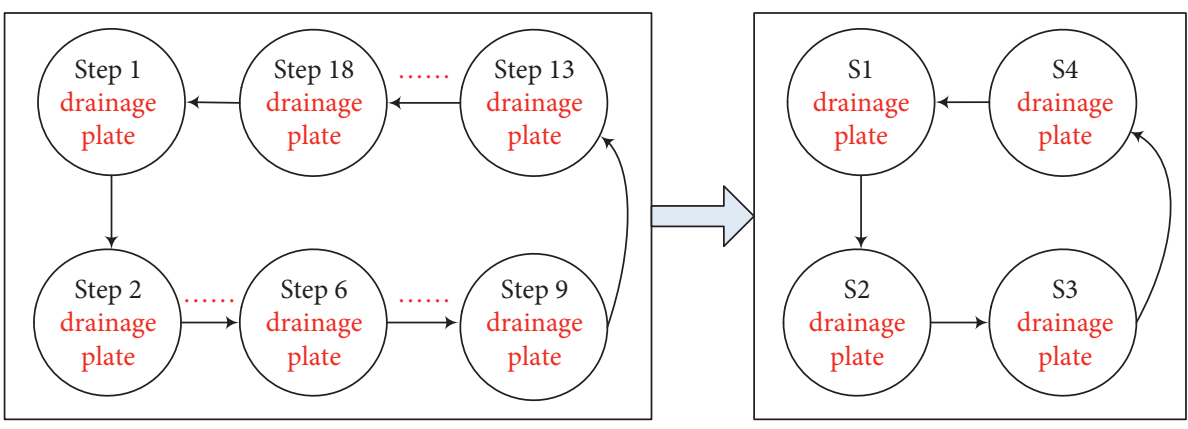

(b)

FIGURE 15: Field autonomous behavior control of drainage plate maintenance robot. (a) Drainage plate operation main process.

(b) Drainage plate operation FSM optimization.

efficiency increased and the robot autonomous behavior ability and intelligence were significantly improved.

It can be seen from Figure 15 that, during the maintenance operation of the drainage plate, a series of state and behavior transitions smoothly from the robot nut alignmentbolt head alignment-nut-fixing bolt head, and the robot drainage plate fastening operation is successfully completed. Due to the intelligent behavior control, the actual operation steps of drainage plate tightening have reduced from eighteen steps of FSM theoretical model to four; taking the average statistical data, the robot operation efficiency has increased and the robot autonomous behavior especially the bolts autonomous capture and positioning on the drainage plate by the end sleeve of the manipulator is much more accurate.
It can be seen from Figure 16 that, in the process of replacement of the damper, a series of state and behavior transitions smoothly by bolt alignment-tightening the boltline clamp alignment-line clamp clamping, and the robot damper replacement operation is successfully completed. Due to the intelligent behavior control, the actual operation steps of damper replacement have reduced from nineteen steps of FSM theoretical model to four. Taking the average statistical data, according to the average of the statistical data, the robot operation efficiency has increased and the robot autonomous behavior, especially the holding mechanism of the old damper and the alignment and positioning control of the damper bolts, has been significantly improved. 


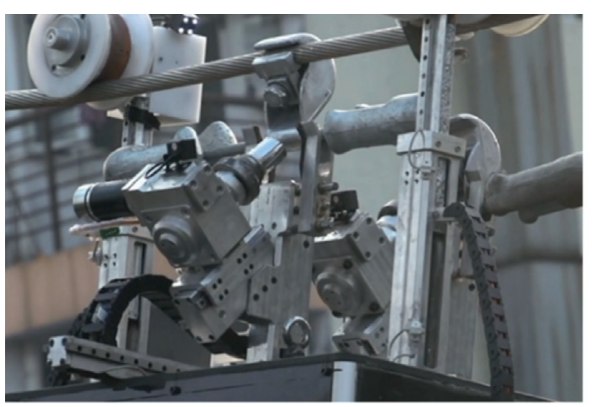

$\mathrm{S}_{1}$ : bolt alignment

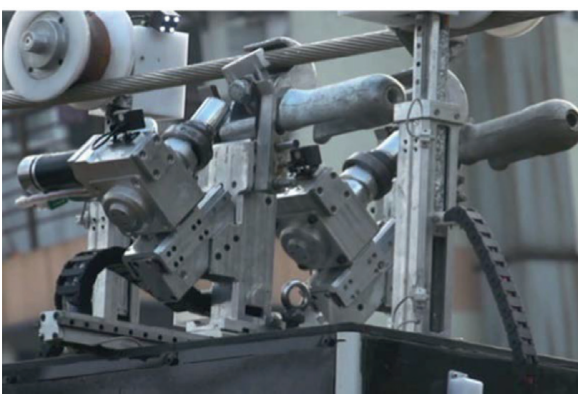

$\mathrm{S}_{3}$ : clamp alignment

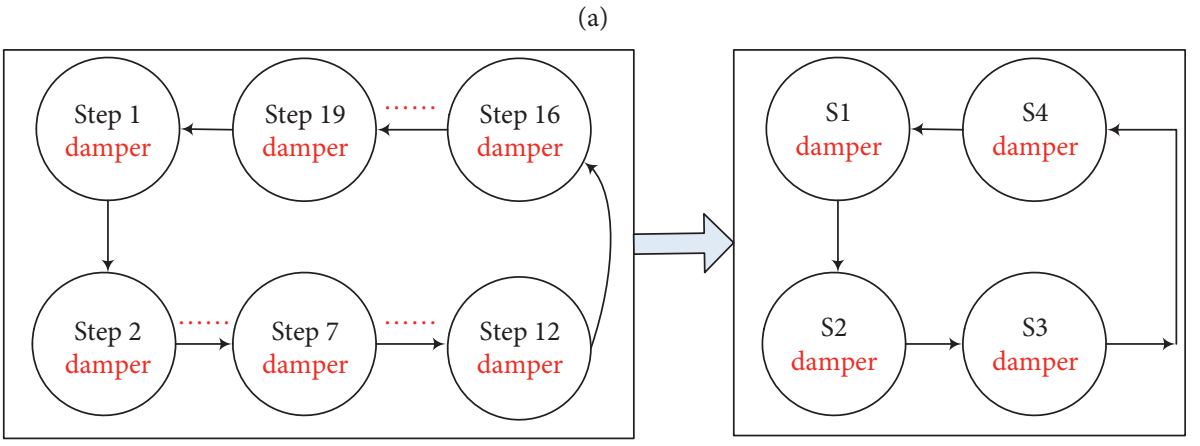

(b)

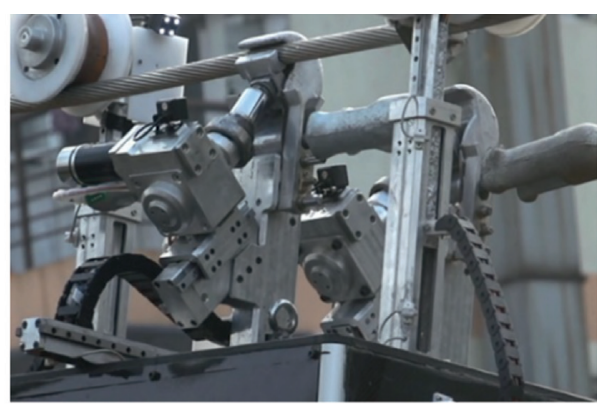

$\mathrm{S}_{2}$ : tightening the bolt

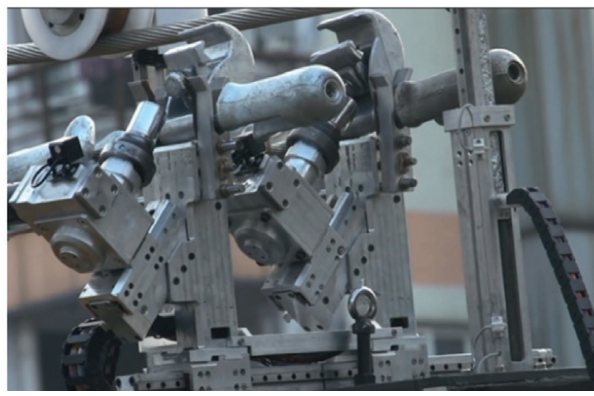

$\mathrm{S}_{4}$ : clip clamping

(a)

FiguRE 16: Field experiment of autonomous behavior control for damper replacement robot. (a) Damper operation main process.

(b) Damper operation FSM optimization.

\section{Conclusion and Future Work}

\subsection{Conclusion}

(1) According to the maintenance requirements of transmission line insulators, drainage plates, and dampers, the basic configuration of the reconfigurable mobile robot at the end of the wheel-arm compound that travels along the transmission line has been designed, and the corresponding operation motion planation has been proposed and developed; it is suitable for the physical prototype of the robot of $220 \mathrm{kV}$ live line.

(2) The robot operation motion behavior can be divided into three categories which are joint behavior, operation arm behavior, and robot behavior. The robot finite state machine model for three different tasks has been designed and a hierarchical architecture, finite state machine model of the robot autonomous behavior control method, has been proposed.
(3) Using the designed FSM model and the robot autonomous behavior control method, three operations have been performed on the $220 \mathrm{kV}$ line with insulators, drainage plate tightening, and damper maintenance. The experimental results show that the autonomous behavior control method can effectively improve robot performance intelligence and operation efficiency.

6.2. Future Work. The live working robot and its highvoltage transmission line operation environment constitute a complex rigid-flexible coupling; in order to meet the basic needs of complex, changeable line environment and practical application, there are still much research that needs to be carried out, such as autonomous behavior control methods. The robustness of the line environment, structural parameters, internal and external disturbances, and uncertain factors still needs further research, the quantified processing method of disturbances and uncertain factors in 
autonomous behavior motion control, the dynamic modeling of robots in a flexible operation environment, and its parameter identification methods. The breakthrough in these key technologies is the key to further improve the robot operation intelligence.

\section{Data Availability}

The experiment data used to support the findings of this study are included within the article.

\section{Conflicts of Interest}

The authors declare that there are no conflicts of interest regarding publishing this manuscript.

\section{Acknowledgments}

This work was supported by the 2020 Opening Fund for Hubei Key Laboratory of Digital Textile Equipment (DTL2020010) and 2020 Intelligent Live Working Technology and Equipment (Robot) Hunan Province Key Laboratory Opening Fund (2020KZD1001).

\section{References}

[1] X. Zheng, G. Wu, W. Jiang, F. Fan, and J. Zhu, "Rigid-flexible coupling dynamics with contact estimator for robot/PTL system," Proceedings of the Institution of Mechanical Engineers, Part K: Journal of Multi-Body Dynamics, vol. 234, no. 4, p. $635,2020$.

[2] N. Pouliot, P.-L. Richard, and S. Montambault, "LineScout technology opens the way to robotic inspection and maintenance of high-voltage power lines," IEEE Power and Energy Technology Systems Journal, vol. 2, no. 1, pp. 1-11, 2015.

[3] M. Wang, G. Wu, F. Fan, Q. Ji, W. He, and Q. Cao, "Dynamic network topology control of branch-trimming robot for transmission lines," Electronics, vol. 8, no. 5, p. 549, 2019.

[4] N. Pouliot, D. Mussard, and S. Montambault, "Localization and archiving of inspection data collected on power lines using LineScout technology," in Proceedings of the Applied Robotics for the Power Industry (CARPI), pp. 197-202, Zurich, Switzerland, September 2012.

[5] K.-H. Seok and Y. S. Kim, "A state of the art of power transmission line maintenance robots," Journal of Electrical Engineering and Technology, vol. 11, no. 5, pp. 1412-1422, 2016.

[6] X. Ye, G. Wu, F. Fan, X. Peng, and K. Wang, "Overhead ground wire detection by fusion global and local features and supervised learning method for a cable inspection robot," Sensor Review, vol. 38, no. 3, pp. 376-386, 2018.

[7] A. B. Alhassan, X. Zhang, H. Shen, and H. Xu, "Power transmission line inspection robots: a review, trends and challenges for future research," International Journal of Electrical Power \& Energy Systems, vol. 118, p. 105862, 2020.

[8] M. F. A. Jalal, K. S. M. Sahari, H. M. Fei, and J. C. T. Leong, "Design and development of three arms transmission line inspection robot," Journal of Robotics, Networking and Artificial Life, vol. 5, no. 3, pp. 157-160, 2018.

[9] H. Kalani, M. Malayjerdi, and M. Hasanpour Dehnavi, "H2M robot: a new prototype robot for insulation of high voltage transmission," International Journal of Intelligent Robotics and Applications, vol. 3, no. 1, pp. 87-98, 2019.
[10] W. Haitao, Y. Biwu, P. Ziheng et al., "Optimal design and stress analysis of the transmission line inspection robot along the ground line," The Journal of Engineering, vol. 2019, no. 16, pp. 3088-3091, 2019.

[11] C. M. Shruthi, A. P. Sudheer, and M. L. Joy, "Optimal crossing and control of mobile dual-arm robot through tension towers by using fuzzy and Newton barrier method," Journal of the Brazilian Society of Mechanical Sciences and Engineering, vol. 41, no. 6, pp. 245-270, 2019.

[12] A. B. Alhassan, X. Zhang, H. Shen et al., "Investigation of aerodynamic stability of a lightweight dual-arm power transmission line inspection robot under the influence of wind," Mathematical Problems in Engineering, vol. 2019, Article ID 2139462, , 2019.

[13] M. Z. Ayyildiz and K. Etinkaya, "Comparison of four different heuristic optimization algorithms for the inverse kinematics solution of a real 4-DOF serial robot manipulator," Neural Computing \& Applications, vol. 27, no. 4, pp. 825-836, 2016.

[14] J. M. S. T. Motta, C. H. Llanos-Quintero, and R. C. Sampaio, "Inverse kinematics and model calibration optimization of a five-DOF robot for repairing the surface profiles of hydraulic turbine blades," International Journal of Advanced Robotic Systems, vol. 13, no. 114, 2016.

[15] C. Sun, W. He, and J. Hong, "Neural network control of a flexible robotic manipulator using the lumped spring-mass model," IEEE Transactions on Systems, Man, and Cybernetics: Systems, vol. 47, no. 8, pp. 1863-1874, 2017.

[16] K. Wang, M. Luo, T. Mei, X. Lin, and Y. Cao, "Posture error correction of a six-DOF serial manipulator based on genetic algorithms," Lecture Notes in Electrical Engineering, vol. 226, no. 5, pp. 203-211, 2013.

[17] M. Moness and A. M. Moustafa, "Tuning a digital multivariable controller for a lab-scale helicopter system via simulated annealing and evolutionary algorithms," Transactions of the Institute of Measurement and Control, vol. 37, no. 10, pp. 1254-1273, 2015.

[18] X. Wu, J. Lin, K. Zhang, and M. Cheng, "Optimal impulsive control for advertising strategy problems based on a gradientbased PSO algorithm," Transactions of the Institute of Measurement and Control, vol. 41, no. 8, pp. 2280-2292, 2019.

[19] H. J. Li, W. Jiang, Y. D. Yan, and W. Chen, "Robust motion control for multi-split transmission line four-wheel driven mobile operation robot in extreme power environment," Industrial Robot: The International Journal of Robotics Research and Application, vol. 47, no. 2, pp. 219-229, 2020.

[20] H. J. Zhang, W. Jiang, and A. Y. Zhang, "Operation motion planning and principle prototype design of four-wheel-driven mobile robot for high-voltage double-split transmission lines," Mathematical Problems in Engineering, vol. 2020, no. 2, 17 pages, Article ID 6195320, 2020.

[21] Z.-b. Zuo and R. Yi, "Obstacle-navigation control of inspection robot for power transmission lines based on knowledge base," Computer Engineering and Applications, vol. 44, no. 3, pp. 236-239, 2008.

[22] E. J. Lima, M. H. S. Bomfim, and M. A. d. M. Mourão, "POLIBOT-Power lines inspection RoBOT," Industrial Robot: An International Journal, vol. 45, no. 1, pp. 98-109, 2018.

[23] W.-bin Guo, H.-g. Wang, Y. Jiang et al., "Obstacle navigation planning for a power transmission line inspection robot]," Robot, vol. 34, no. 4, pp. 505-512, 2012.

[24] G. Tao and L. Fang, "A multi-unit serial inspection robot for power transmission lines," Industrial Robot: The International Journal of Robotics Research and Application, vol. 46, no. 2, pp. 223-234, 2019. 
[25] S. Yi-feng, H.-g. Wang, Li Zhen-hui et al., "Vision based transmission line broken strand detection and robot behavior planning," Robot, vol. 37, no. 2, pp. 204-211, 2015.

[26] Y. Jiang, H.-g. Wang, and F. Li-jin, "Gait control of micro wall-climbing robot based on initiative exploration," Journal of Mechanical Engineering, vol. 45, no. 07, pp. 56-62, 2009.

[27] W. Wang, G.-p. Wu, Y.-c. Bai et al., "Hand-eye-vision based control for an inspection robot's autonomous line grasping," Journal of Central South University, vol. 21, no. 6, pp. 22162227, 2014.

[28] W. Wang, Y. Bai, G. Wu et al., "An electromagnetic navigation method for line-following robot based on information fusionJ," Automation of Electric Power Systems, vol. 37, no. 16, pp. 73-79, 2013.

[29] Y. Yan, W. Jiang, A. Zhang et al., "Research on configuration design and operation effect evaluation for ultra high voltage (UHV) vertical insulator cleaning robot," Industrial Robot: The International Journal of Robotics Research and Application, vol. 47, no. 1, pp. 90-101, 2019.

[30] W. Jiang, A. Zhang, G. Wu et al., "Manipulator visual localization motion control for power cable mobile robot in dynamic-unstructured environment," Industrial Robot: The International Journal of Robotics Research and Application, vol. 46, no. 1, pp. 93-103, 2019.

[31] W. Jiang, G. Wu, F. Fan et al., "Structure singular value theory based robust motion control of live maintenance robot with reconfigurable terminal function for high voltage transmission line," International Journal of Advanced Robotic Systems, vol. 15, no. 2, pp. 1-13, 2018. 